\section{Absaugsysteme für die dentale Chirurgie}

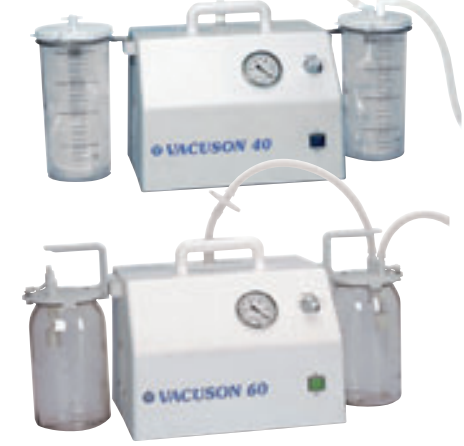

Konventionelle zahnärztliche Saugsysteme haben neben der Entfernung von Speichel und Sekreten aus dem Mund des Patienten eine wichtige und zentrale Aufgabe: die Absaugung des kontaminierten Spraynebels (Aerosol) aus dem gesamten Behandlungsumfeld. Dafür ist ein großes Saugvolumen von ca. 300 I/min bei einem niedrigen Unterdruck von ca. 120 mbar erforderlich.

Anders verhält es sich in der Oral- und MKG-Chirurgie. Während der Operation müssen punktgenau größere Blutmengen, Knochen-, Gewebe- und Dentinreste mithilfe der chirurgischen Kanüle entfernt werden, was einen größeren Unterdruck erfordert. Einsätze mit herkömmlichen Saugsystemen führen meist durch Verstopfungen zu Beschädigungen und verursachen so vermeidbare Kosten.

Um die gesamte Konzentration auf den Patienten gewährleisten zu können, muss sich das Behandlerteam voll und ganz auf reibungslos funktionierende Systeme verlassen können.

Die mobilen Saugsysteme Vacuson 40 und Vacuson 60 der Firma NOUVAG bieten eine maßgeschneiderte Lösung für alle Erfordernisse der dentalen Chirurgie.

Die Dauerlauffähigkeit, eine Saugleistung von 40 1/min sowie $60 \mathrm{l} / \mathrm{min}$ und ein konstant hoher Unterdruck (stufenlos regelbar) von bis zu 0,9 bar/676,7 mmHG garantieren ein freies Operationsfeld.

Durch die bewährte 1- bzw. 2-KolbenZylinder-Technologie wird ein schneller Vakuumaufbau und eine hohe Saugleistung bei leisem und vibrationsarmen Betrieb erzielt.

Sterilisierbare Behälter, als Ein- oder Mehrwegsysteme (2 1/5 l) erhältlich, bieten eine einfache, hygienische und sichere Sekretentsorgung. Ein mechani-

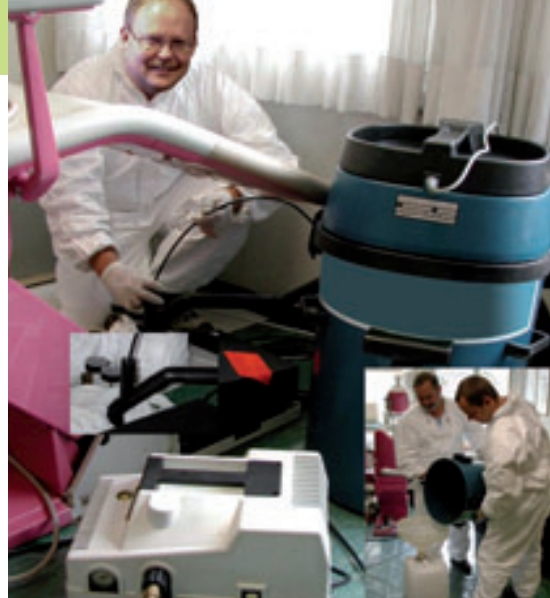

sches Sicherheitsventil im Flaschendeckel verhindert ein Überlaufen des Sekrets. Die semipermeable Membran des Bakterienfilters schließt bei Kontakt mit Flüssigkeiten und verhindert ein Eindringen in das Gerät, wodurch ein weiterer zuverlässiger Überlaufschutz gegeben ist. Ebenso verhindert der Filter weitestgehend das Eindringen von Bakterien und Kleinstteilchen und schützt somit die Vakuumpumpe, aber auch das Betriebsumfeld gegen eine Kontamination. Die desinfektionbeständigen Geräteoberflächen runden die Hygieneanforderungen ab.

Aufgrund des geringen Gewichts und der kompakten Ausführung sowie den optional erhältlichen Stativen ist das System flexibel und funktionell einsetzbar.

\section{NOUVAG GmbH}

Schulthaißstrasse15

78462 Konstanz

E-Mail: info-de@nouvag.com

Internet: www.nouvag.com 